\title{
Abundances in Stars with Exoplanets
}

\author{
Garik Israelian \\ Instituto de Astrofísica de Canarias, E-38205 La Laguna, \\ Tenerife, Spain \\ e-mail: gil@iac.es
}

\begin{abstract}
Extensive spectroscopic studies of stars with and without planets have concluded that stars hosting planets are significantly more metal-rich than those without planets. More subtle trends of different chemical elements begin to appear as the number of detected extrasolar planetary systems continues to grow. I review our current knowledge concerning the observed abundance trends of various chemical elements in stars with exoplanets and their possible implications.
\end{abstract}

\section{Introduction}

Beginning with the discovery by Mayor \& Queloz (1995) of a giant planet racing around 51 Pegasi, the number of planets orbiting solar-type stars has now reached 115. Most of the planets have been discovered by the Geneva and California \& Carnegie groups led by Michel Mayor (Geneva), Geoff Marcy (California) and Paul Butler (Carnegie). This sample size is now sufficient to search for various trends linking the properties of exoplanets and those of their parent stars. It has been suggested that one of the key factors relevant to the mechanisms of planetary system formation is the amount of metals available in proto-planetary discs.

The chemical abundance studies of planet hosts are based on high signalto-noise $(\mathrm{S} / \mathrm{N})$ and high resolution spectra. Many targets have been observed by more than one group, allowing useful cross checks of their analyses and spectra. Most chemical studies of the planet hosts used iron as the reference element (Gonzalez 1997, Laws et al. 2003, Murray \& Chaboyer 2002, Santos, Israelian \& Mayor 2001, 2003, Santos et al. 2003b) and only a few studies have discussed the abundance trends of other metals (Gonzalez \& Laws 2000, Sadakane et al. 2003, Gonzalez et al. 2001, Santos, Israelian \& Mayor 2000, Bodaghee et al. 2003). The authors in most of these studies have been constrained to compare the results for the planet host sample with other studies in the literature. However, in some articles such a comparison was not provided, leaving room for any kind of speculation regarding the source of the abundance anomalies. Different authors used different sets of lines, atmospheric parameters, data, etc. These are all potential sources of systematic error. To overcome this problem, Santos et al. (2001) prepared a sample of stars without known planets. To ensure a high degree of consistency between the two samples, these stars were analyzed and observed in the same way as the planet hosts. Further spectroscopic analysis by 
Santos et al. (2003a, 2003b), Israelian et al. (2003) and Bodaghee et al. (2003) were based on this same comparison sample.

Chemical abundance studies of planet host stars have revealed that their metallicities are higher on average than those typically found among solar-type disk stars without known planets (Gonzalez 1997, Laws et al. 2003, Santos et al. 2001, 2003a, 2003b). In Fig. 1 a comparison between the metallicity distributions for a volume-limited comparison sample of stars and for the 87 planet hosts from Santos et al. (2003a) is presented. The stars with planets are metalrich compared with the comparison sample stars by, on average, 0.25 dex. This suggests that the metallicity and the presence of giant planets are linked. The metallicity excess could result from the accretion of planets and/or planetesimals onto the star (Gonzalez 1997). Opposing this view, Santos et al. (2000, 2001) proposed that the source of the high metallicity is primordial and the observed abundance trends represent those from the proto-planetary and protostellar molecular cloud out of which the star and the planets formed. This idea would support the classical CIA (core-instability accretion) model (Pollack et al. 1996) where some $10-15 \mathrm{M}_{\oplus}$ masses of planetesimals condense into a rocky core. The initial metallicity of the parental cloud is a key parameter in this scheme. Results from Bodaghee et al. (2003) clearly demonstrate that the excess in metallicity observed for planet host stars is widespread and not unique to iron. Abundances of different elements may provide clue for checking various planet formation, and even planet migration, hypothesis. The self-enrichment scenario (Gonzalez 1997) which has been proposed to explain the $[\mathrm{Fe} / \mathrm{H}]$ excess in host stars should lead to a relative overabundance of refractory elements (iron-group, $\alpha$-elements etc.) compared to volatiles (C, N, O, S, Zn). Volatiles are known to condense into solid grains at relatively low temperatures, and are expected to behave differently compared to the refractories which condense at high temperature. If the star accreted a considerable amount of planetary material, then high temperatures near the star would favor the addition of refractory elements over volatiles (which are locked in giant planets) and a trend in abundance versus condensation temperatures may appear (Smith et al. 2001).

\section{Abundances of light elements}

The light elements $\mathrm{Li}$ and $\mathrm{Be}$ are important tracers of the internal structure and pre-main sequence evolution of solar type stars since they provide information regarding the redistribution and mixing of matter within a star. Studies of $\mathrm{Be}$ and $\mathrm{Li}$ complement each other as $\mathrm{Li}$ is depleted at much lower temperatures than $\mathrm{Be}$. By measuring $\mathrm{Li}$ and $\mathrm{Be}$ in stars hosting planets we can obtain crucial information about the mixing, diffusion and angular momentum history of the stars. Accretion of planets and planetesimals, stellar activity and tidal interactions in star-planets systems may largely modify the surface abundances of the light elements.

\subsection{Lithium}

Gonzalez \& Laws (2000) presented a direct comparison of $\mathrm{Li}$ abundances among planet-harbouring stars with field stars without planets and proposed that the former have less Li. However, in a critical analysis of this problem Ryan (2000) 

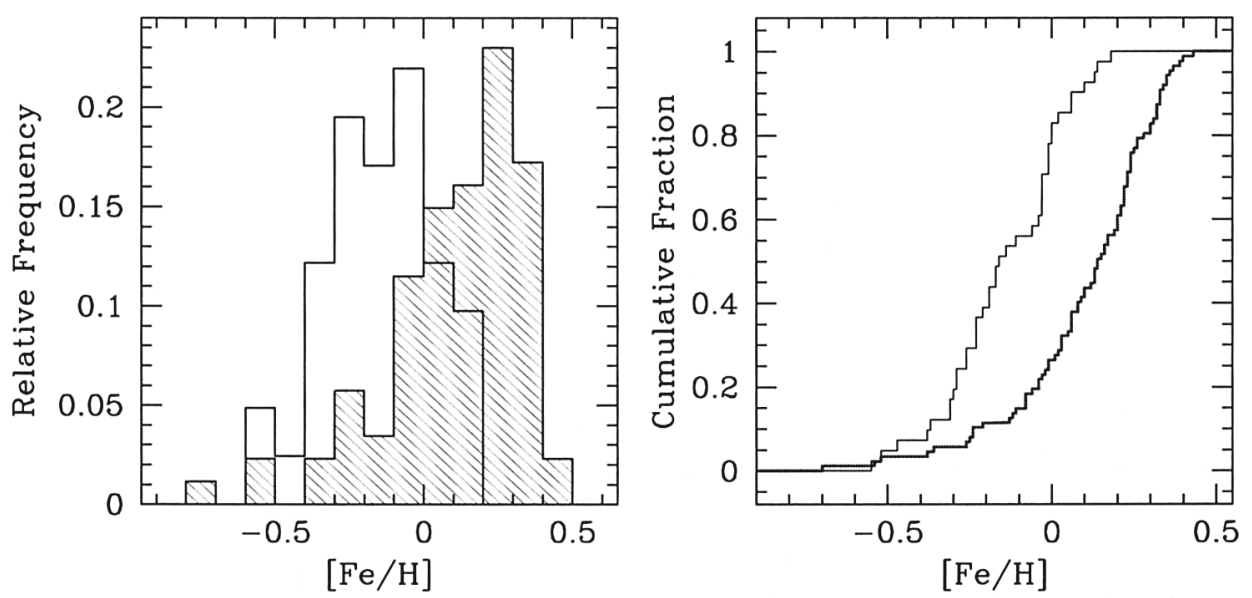

Figure 1. Metallicity distribution for stars with planets (hatched histogram) compared with the same distribution for the field stars without planets (empty histogram). A Kolmogorov-Smirnov test shows the probability for the two populations being part of the same sample to be $10^{-8}$. From Santos et al. (2003a).

concludes that planet hosts and field stars have similar $\mathrm{Li}$ abundances. Given the larger number of planet-harbouring stars, we re-investigated the Li problem (Israelian et al. 2003) and looked for various statistical trends. When the $\mathrm{Li}$ abundances of planet host stars are compared with the 157 field stars in the sample of Chen et al. (2001), we find that the $\mathrm{Li}$ abundance distributions in the two samples are different (Fig. 2). There is a possible excess of Li depletion in planet hosts having effective temperatures in the range $5600-5850 \mathrm{~K}$, whereas we find no significant differences for stars with temperatures in the range 5850-6350 $\mathrm{K}$ (Fig. 3). One may ask, why is the difference only seen in stars with effective temperatures in the range $5600-5850 \mathrm{~K}$ ? Given the depth of the surface convection zone, we expect that any effect on the $\mathrm{Li}$ abundance will be more apparent in solar-type stars. Lower mass stars have deeper convective zones and destroy Li more efficiently, so we can often only set upper limits to the abundance. However, the convective layers of stars more massive than the Sun do not reach the lithium burning layer and therefore these stars generally preserve a large fraction of their original $\mathrm{Li}$. The relatively small dispersion of $\mathrm{Li}$ abundances in these hotter stars is clearly seen in Fig. 3. Therefore it seems that solar-type stars are the best targets for investigating any possible (and maybe marginal) effects of planets on the evolution of the stellar atmospheric abundance of $\mathrm{Li}$.

There are at least two possible hypothesis for the lower $\mathrm{Li}$ abundance in planet-hosting stars. It is possible that proto-planetary disks lock a lot of angular momentum and therefore create some rotational braking in the host stars during their pre main-sequence evolution. The lithium is efficiently destroyed during this process due to an increased mixing. The extra Li depletion can also be associated with a planet migration mechanism at early times in the evolution of the star when the superficial convective layers may have been rotationally de- 

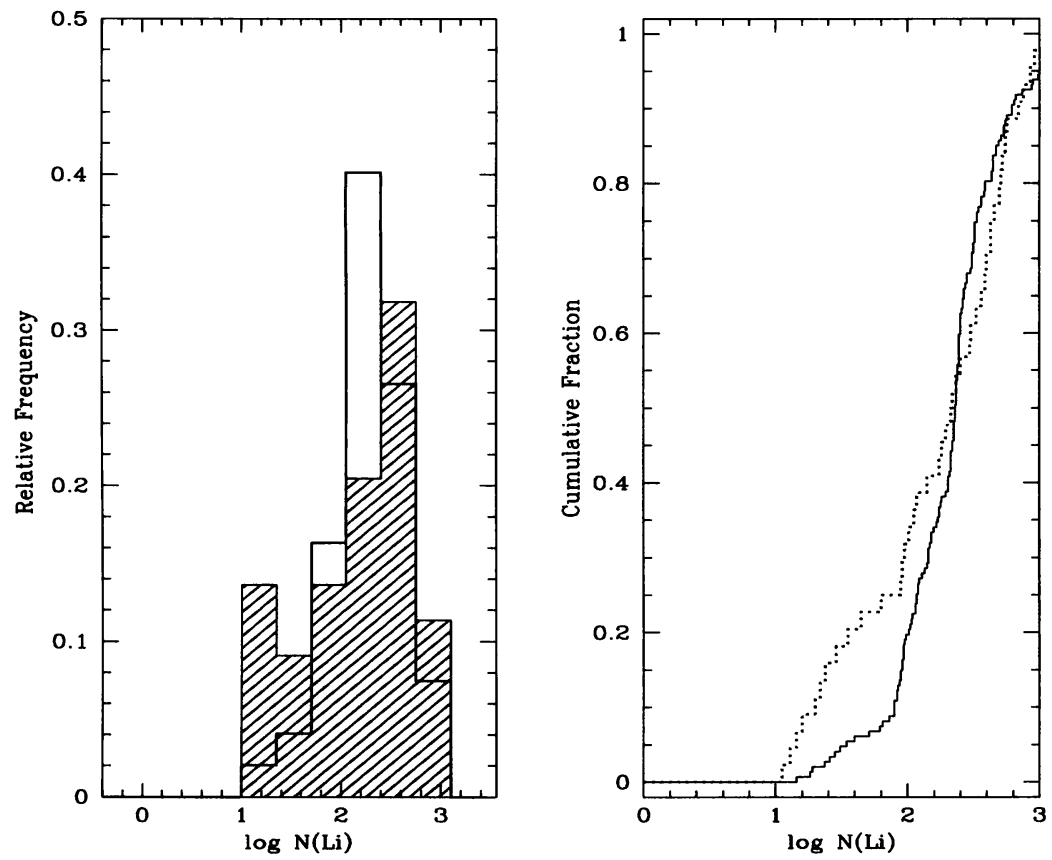

Figure 2. Lithium distribution for stars with planets (hatched histogram) compared with the same distribution for the field stars from Chen et al. 2001. (empty histogram). A Kolmogorov-Smirnov test shows the probability for the two populations being a part of the same sample is 0.2 . From Israelian et al. (2003).

coupled from the interior. Efficient depletion may be caused by a strong mixing due to the migration-triggered tidal forces, which create a shear instability. The mass of the decoupled convection zone in these stars is comparable to the masses of the known exoplanets; therefore, the migration of one or more giant planets could indeed produce an observable effect. The planetary migration may also trigger the accretion of planetesimals, inducing metallicity enhancement. Some fresh $\mathrm{Li}$ could also be added in the convective zone. However, if this process takes place in the early evolution of the star, the freshly added Li will be destroyed.

Observations show a possible lack of short-period massive planets with $M>$ $4 M_{\mathrm{J}}$ orbiting around $\mathrm{Li}$ depleted stars with $1<\log \epsilon(\mathrm{Li})<1.6$ (Israelian et al. 2003). When considering stars with $\log \epsilon(\mathrm{Li})$ between 1.6 and 3 , we find that about half have planets with $M>4 M_{\mathrm{J}}$. There is a clear link between Mass-Li and Period-Li relationships most likely associated with the already proposed correlation between mass and period of planetary companions (Zucker \& Mazeh 2002; Udry et al. 2002).

\subsection{The ${ }^{6} \mathrm{Li}$-test}

A unique opportunity for testing the planet and/or planetesimal accretion scenario is offered by a ${ }^{6} \mathrm{Li}$-test proposed by Israelian et al. (2001). This approach is based on looking for an element that should not appear in the atmosphere 
of a normal solar-type star, but would be present in a star that has accreted planetary matter. Nuclear reactions destroy the ${ }^{6} \mathrm{Li}$ and ${ }^{7} \mathrm{Li}$ isotopes in stellar interiors at temperatures of $2 \times 10^{6}\left({ }^{6} \mathrm{Li}\right)$ and $2.5 \times 10^{6} \mathrm{~K}\left({ }^{7} \mathrm{Li}\right)$. Furthermore, convection cleans the upper atmosphere of $\mathrm{Li}$ nuclei by transporting them to deeper and hotter layers where they are rapidly destroyed. Young solar-type stars are entirely convective and most of the primordial $\mathrm{Li}$ nuclei are burned in their interiors in a mere few million years. However, many solar-type stars preserve a large fraction of their initial atmospheric ${ }^{7} \mathrm{Li}$ nuclei. According to standard models (Forestini 1994), at a given metallicity there is a mass range where ${ }^{6} \mathrm{Li}$, but not ${ }^{7} \mathrm{Li}$, is destroyed. These models predict that no ${ }^{6} \mathrm{Li}$ can survive pre-MS mixing in metal-rich solar-type stars. The detection of ${ }^{6} \mathrm{Li}$ in HD 82943 (Israelian et al. 2001, 2004) is convincing observational evidence that stars may accrete planetary material, or even entire planets, during their main sequence evolution. Other explanations of this phenomenon such as stellar flares or surface spots have been ruled out (Israelian et al. 2001). Sandquist et al. (2002) have recently proposed that ${ }^{6} \mathrm{Li}$ can be used to distinguish between different giant planet formation theories.

However, analysis of ${ }^{6} \mathrm{Li}$ is difficult. First of all, it is a weak component of a blend with much stronger doublet of ${ }^{7} \mathrm{Li}$ having an isotopic separation of $0.16 \AA$. Blending of the $\mathrm{Li}$ line with other weak absorptions and the placement of continuum pose serious problems in metal-rich solar-type stars. Spectra with $\mathrm{S} / \mathrm{N} \sim 1000$ and a resolving power of at least $\lambda / \Delta \lambda \sim 100.000$ are required to tackle these problems. In metal-rich stars the identification of any weak blends in the region of the $\mathrm{Li}$ absorption becomes crucial. For example, Reddy et al. (2002) claimed that a previously noticed weak absorption in the solar spectrum at $6708.025 \AA$ belongs to Ti I. With this assumption their study of the $\mathrm{Li}$ feature in $\mathrm{HD} 82943$ did not confirm the presence of ${ }^{6} \mathrm{Li}$. However, our recent analysis (Israelian et al. 2004) does not support the identification of a weak absorption feature at $6708.025 \AA$ with the low excitation $\mathrm{Ti}$ I line. We have suggested that the unidentified absorption is most probably produced by a high excitation Si I line. The presence of ${ }^{6} \mathrm{Li}$ in $\mathrm{HD} 82943$ was confirmed with the updated value for the isotopic ratio $f\left({ }^{6} \mathrm{Li}\right)=0.05 \pm 0.02$ by taking the $\mathrm{Si}$ I line into account in the reanalysis of the ${ }^{6} \mathrm{Li} /{ }^{7} \mathrm{Li}$ and using new VLT/UVES spectra with $\mathrm{S} / \mathrm{N} \sim 1000$.

Slow accretion of planetesimals was invoked in order to explain the $[\mathrm{Fe} / \mathrm{H}]$ distribution in planet-harbouring stars. Recently, Murray \& Chaboyer (2002) concluded that an average of $6.5 M_{\oplus}$ of iron must be added to the planetharbouring stars in order to explain the mass-metallicity and age-metallicity relations. Accretion of $6.5 M_{\oplus}$ of planetesimals of iron during early MS evolution will strongly modify ${ }^{7} \mathrm{Li}$ abundances in these stars. Moreover, given the depth of the convection zone in stars with $\mathrm{T}_{\text {eff }}>5900 \mathrm{~K}$, a large amount of the added ${ }^{6} \mathrm{Li}$ may avoid destruction via mixing. Accretion of a chondritic matter with $6.5 M_{\oplus}$ of iron by a star with $T_{\text {eff }}=6100 \mathrm{~K}$ and with a convection zone mass $10^{-3} \mathrm{M}_{\odot}$ will rise its ${ }^{7} \mathrm{Li}$ abundance from $\log \epsilon(\mathrm{Li})=2.7$ to 3.2 while the isotopic ratio will become $f\left({ }^{6} \mathrm{Li}\right)=0.06$. This will create a detectable ${ }^{6} \mathrm{Li}$ absorption feature with an equivalent width (EW) $\sim 4 \mathrm{~m} \AA$. This feature can be measured even if it is blended with the line at $6708.025 \AA$ because the latter is expected 

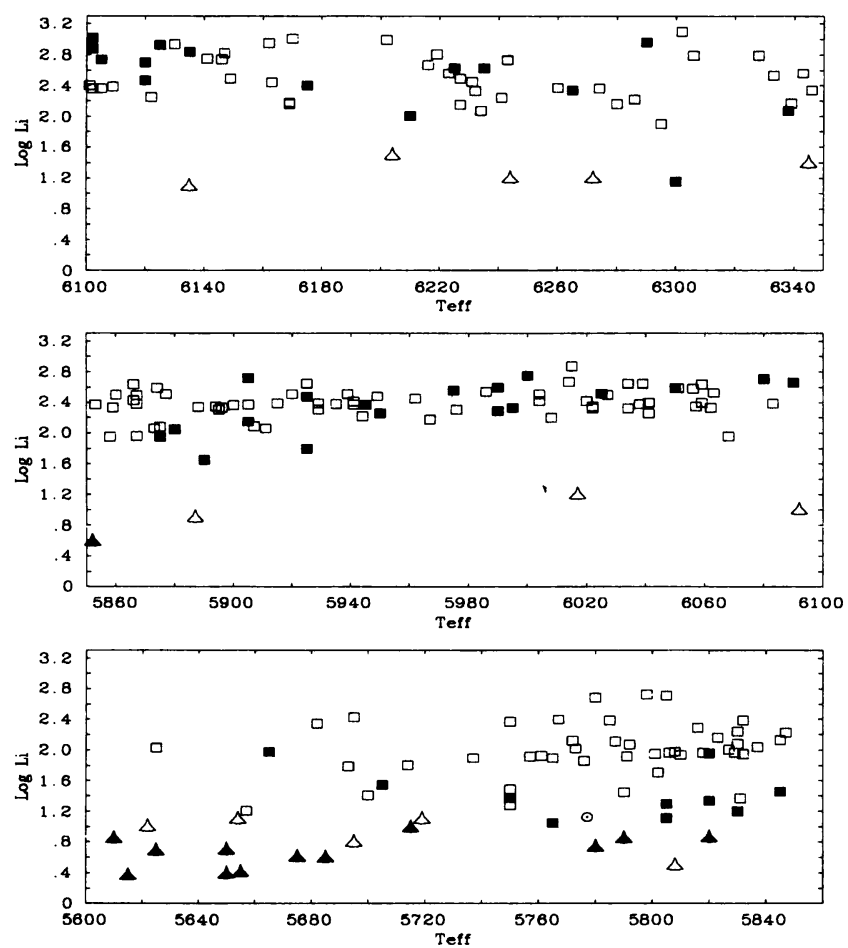

Figure 3. Lithium versus effective temperature for stars with planets (filled squares) and the comparison sample of Chen et al. (2001) (empty squares). Upper limits are filled (planet hosts) and empty (comparison sample) triangles. The position of the Sun is indicated. From Israelian et al. (2003).

to appear with an EW $\lesssim 2 \mathrm{~m} \AA$ in these type of stars (no matter which kind of element/line is responsible for this absorption).

\subsection{Beryllium}

The first studies of Be in planet hosts were those by García López \& Pérez de Taoro (1998) and Deliyannis et al. (2000). However, these authors did not arrive to any firm conclusion because of the lack of a comparison sample of stars and the low number of planet hosts in their studies. Santos et al. (2002) derived beryllium abundances for a sample of 29 planet host and 6 "single" stars aimed at studying in detail the effects of the presence of planets on the structure and evolution of the associated stars. Their preliminary results suggest that theoretical models may have to be revised for stars with $\mathrm{T}_{\text {eff }}<5500 \mathrm{~K}$. Santos et al. (2002) found several Be depleted stars at $5200 \mathrm{~K}$ which current models cannot explain. A comparison between planet-hosting stars and "single" stars (although very few in their analysis) shows no clear difference between either population. Their preliminary result supports a "primordial" origin for 
the metallicity excess observed in the planet hosts. The analysis of a comparison sample will certainly help to further constrain the models.

\section{Abundances of metals}

\subsection{Volatiles}

Santos et al. (2000) and Gonzalez \& Laws (2000) have noticed that planet hosts tend to show subsolar $[\mathrm{C} / \mathrm{Fe}]$ with increasing $[\mathrm{Fe} / \mathrm{H}]$. Gonzalez et al. (2001) were less certain about these findings, while Takeda et al. (2001) and Sadakane et al. (2002) found no clear evidence of the constant $[\mathrm{C} / \mathrm{Fe}]$ in the metallicity range $-0.5 \lesssim[\mathrm{Fe} / \mathrm{H}] \lesssim 0.4$. However, all these authors used field stars from the literature to build up their small and inhomogeneous comparison samples. For example, Sadakane et al. (2002) used for C and O the results of the analysis by Gustaffson et al. (1999), Edvardsson et al. (1993), Chen et al. (2000) and Feltzing \& Gustaffson (1998). They concluded that $[\mathrm{C} / \mathrm{Fe}]$ and $[\mathrm{O} / \mathrm{Fe}]$ ratios in planet hosts closely follow the trends observed in field stars with $[\mathrm{C} / \mathrm{Fe}]=[\mathrm{O} / \mathrm{Fe}]=0$ at $[\mathrm{Fe} / \mathrm{H}]>0$. Although $\mathrm{N}$ abundances were derived by Gonzalez \& Laws (2000), Gonzalez et al. (2001) and Sadakane et al. (2002), none of these authors discussed the trends of $[\mathrm{N} / \mathrm{Fe}]$ and $[\mathrm{N} / \mathrm{H}]$.

Recently, Ecuvillon et al. (2003) used VLT/UVES spectra in order to derive the $\mathrm{N}$ abundance in a large number of planet hosting stars and comparison sample stars from Santos et al. (2001). The near-UV NH band at 3340-3380 was employed in their analysis. In addition, they studied $\mathrm{C}$ and $\mathrm{S}$ abundances from several optical lines. Their results indicate a clear difference in $[\mathrm{N} / \mathrm{H}],[\mathrm{C} / \mathrm{H}]$ and $[\mathrm{S} / \mathrm{H}]$ distributions for both samples. They found that $[\mathrm{S} / \mathrm{Fe}]$ and $[\mathrm{N} / \mathrm{Fe}]$ are flat at $[\mathrm{Fe} / \mathrm{H}]>0$. The final abundance ratios of $\mathrm{C}, \mathrm{N}$ and $\mathrm{S}$ for both samples as functions of $[\mathrm{Fe} / \mathrm{H}]$ are displayed in Fig. 4. The trend of $[\mathrm{C} / \mathrm{Fe}]$ is similar to the $[\mathrm{O} / \mathrm{Fe}]$ trend; monotonically decreasing at higher $[\mathrm{Fe} / \mathrm{H}]$, as discussed by Bensby et al. (2003) and Feltzing \& Gustaffson (1998). Finally we note a small increase in $[\mathrm{S} / \mathrm{Fe}]$ at $[\mathrm{Fe} / \mathrm{H}]>0.2$ (see Fig. 4) also seen in the Fig. 10 of Sadakane et al. (2002). We cannot rule out the possibility that the $[\mathrm{S} / \mathrm{H}]$ ratios of planet-bearing stars are not an iron-rich extension to the $[\mathrm{S} / \mathrm{H}]$ trends of the comparison sample. The behaviour of $[\mathrm{X} / \mathrm{H}]$ versus $[\mathrm{Fe} / \mathrm{H}]$ for some volatiles coupled with the presence of giant planets merits further investigation.

\subsection{Refractories}

The first abundance studies of several refractory elements (Gonzalez 1997, Gonzalez \& Laws 2000, Gonzalez et al. 2001, Santos et al. 2000) revealed a few possible anomalies. Gonzalez et al. (2001) claimed that the stars with planets appear to have smaller $[\mathrm{Na} / \mathrm{Fe}],[\mathrm{Mg} / \mathrm{Fe}]$ and $[\mathrm{Al} / \mathrm{Fe}]$ values than field dwarfs of the same $[\mathrm{Fe} / \mathrm{H}]$. These authors did not find any significant differences for the refractories $\mathrm{Si}, \mathrm{Ca}$ and $\mathrm{Ti}$. However, the abundance trends in the few planet hosts discussed by Takeda et al. (2001) and Sadakane et al. (2002) did not show anything peculiar. On the other hand, anomalies were found by Sadakane et al. (2003) who detected a few planet-bearing stars with an interesting abundance pattern in which the volatile elements $\mathrm{C}$ and $\mathrm{O}$ are underabundant with respect to refractories $\mathrm{Si}$ and $\mathrm{Ti}$. 

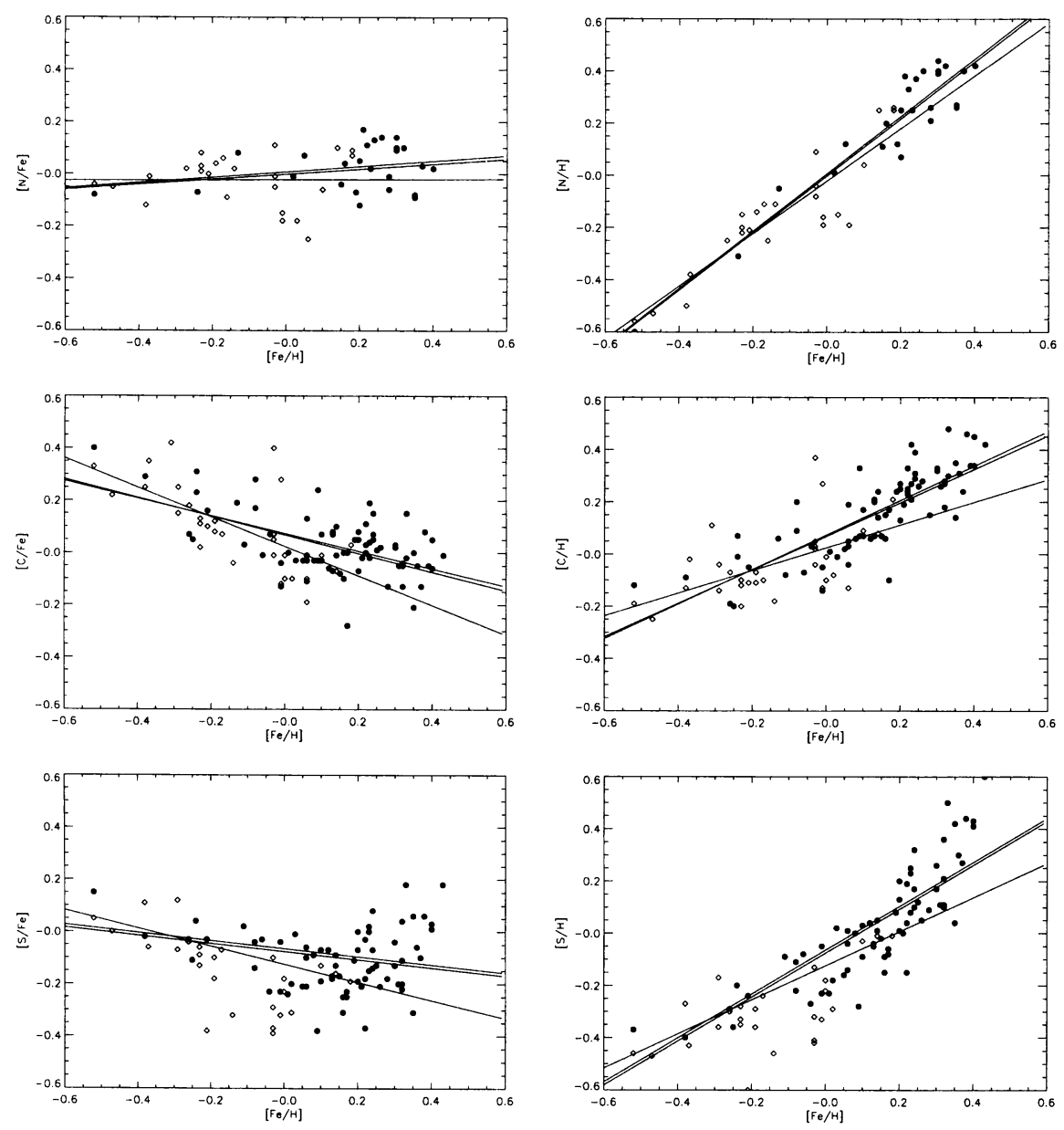

Figure 4. $[\mathrm{X} / \mathrm{Fe}]$ vs. $[\mathrm{Fe} / \mathrm{H}]$ plots (left) and $[\mathrm{X} / \mathrm{H}]$ vs. $[\mathrm{Fe} / \mathrm{H}]$ plots (right) for N, C and S. Filled circles represent planet host stars, while open symbols denote comparison sample stars. Linear least-square fits to the planet hosts (dotted lines), to the comparison sample stars (dashed lines) and to both samples together (solid lines) are represented. Only the stars with $T_{\text {eff }}>5200 \mathrm{~K}$ were considered in the analysis of S. From Ecuvillon et al. (2003). 
A uniform and unbiased comparison of abundances of some $\alpha$ - (Si, Ca, $\mathrm{Ti}$ ) and Fe-group ( $\mathrm{Sc}, \mathrm{V}, \mathrm{Cr}, \mathrm{Mn}, \mathrm{Co}, \mathrm{Ni}$ ) elements in 77 planet host and 42 comparison sample stars without planets was carried out by Bodaghee et al. (2003). These authors concluded that the abundance trends for the planet hosts are almost identical to those in the field. Slight differences were found for $\mathrm{V}, \mathrm{Mn}$ and, to a lesser extent, Co and Ti (Fig. 5). Although the abundance scatter for most of the elements was found to be small, a few elements showed considerable dependence of the derived abundances on the effective temperature. The largest effect was found for Ti, Co and V, where the difference between $\mathrm{K}$ and F-dwarfs has reached 0.2-0.3 dex. These trends might be related to NLTE effects.

In general, the abundance distributions of planet host stars are high $[\mathrm{Fe} / \mathrm{H}]$ extensions to the curves traced by the field dwarfs without planets. No significant differences are found in the regions of overlap. However, although some differences for certain elements are subtle (and may even be negligible), they are certainly intriguing enough to merit additional studies.

\section{Implications}

\subsection{Chemical Evolution of the Galaxy}

One of the byproducts of chemical abundance studies in planet-hosting stars is the possibility of learning about Galactic chemical evolution trends at high metallicities. The number of detailed abundance studies at $[\mathrm{Fe} / \mathrm{H}]>0$ is very limited and exoplanet hosts can help to explore this regime. Some of the trends obtained in these studies may be linked with the presence of giant planets. According to Santos et al. (2003a), more than $25 \%$ of stars with $[\mathrm{Fe} / \mathrm{H}]>0.3$ host planets. The possibility that all metal-rich stars host planetary systems cannot be ruled out. Thus, it is almost impossible to compare stars with and without planets in the high $[\mathrm{Fe} / \mathrm{H}]$ tail of the distribution. The relative frequency of stars with planets increases with $[\mathrm{Fe} / \mathrm{H}]$, but there is a sharp cutoff once the metallicity reaches about 0.4 dex (Fig. 1). It is hard to believe that Nature could somehow tune the pollution process (i.e. self-enrichment) in planet hosts by not allowing them to have $[\mathrm{Fe} / \mathrm{H}]>0.5$. Most probably the cutoff represents a rough upper limit to metallicities in the solar neibourhood. If $[\mathrm{Fe} / \mathrm{H}] \sim 0.4$ represents the "present day" state of Galactic chemical evolution, then certain trends should appear for all other chemical species. How then can we disentangle the abundance anomalies produced by the presence of planets ?

The most simple way is to study those trends which are difficult to interpret in the framework of standard Galactic chemical evolution models. Given the constant rate of Type II and Type Ia SN during the last 10 Gyrs of Galactic evolution, we would not expect any significant change in the slope of $[\alpha / \mathrm{Fe}]$ versus $[\mathrm{Fe} / \mathrm{H}]$ where the $\alpha$ 's are: $\mathrm{O}, \mathrm{Si}, \mathrm{S}, \mathrm{Mg}, \mathrm{Ca}$ and $\mathrm{Ti}$. However, observations (Gonzalez et al. 2001, Sadakane et al. 2002, Bodaghee et al. 2003) show a sudden change at $[\mathrm{Fe} / \mathrm{H}]=0$ in the slopes of $[\mathrm{Si} / \mathrm{Fe}]$ and $[\mathrm{Ti} / \mathrm{Fe}]$ versus $[\mathrm{Fe} / \mathrm{H}]$ while $[\mathrm{Ca} / \mathrm{Fe}]$ decreases monotonically with $[\mathrm{Fe} / \mathrm{H}]$. It is not clear why $\mathrm{Si}$ and $\mathrm{Ti}$ should drastically change their slopes at $[\mathrm{Fe} / \mathrm{H}]=0$ and become flat. Moreover, high quality observations by Bensby et al. (2003) demonstrate that $[\mathrm{O} / \mathrm{Fe}]$ continues to decrease at $[\mathrm{Fe} / \mathrm{H}]>0$ without showing the flattening out found in previous studies (Nissen \& Edvardsson 1992). Galactic chemical evolution models predict 

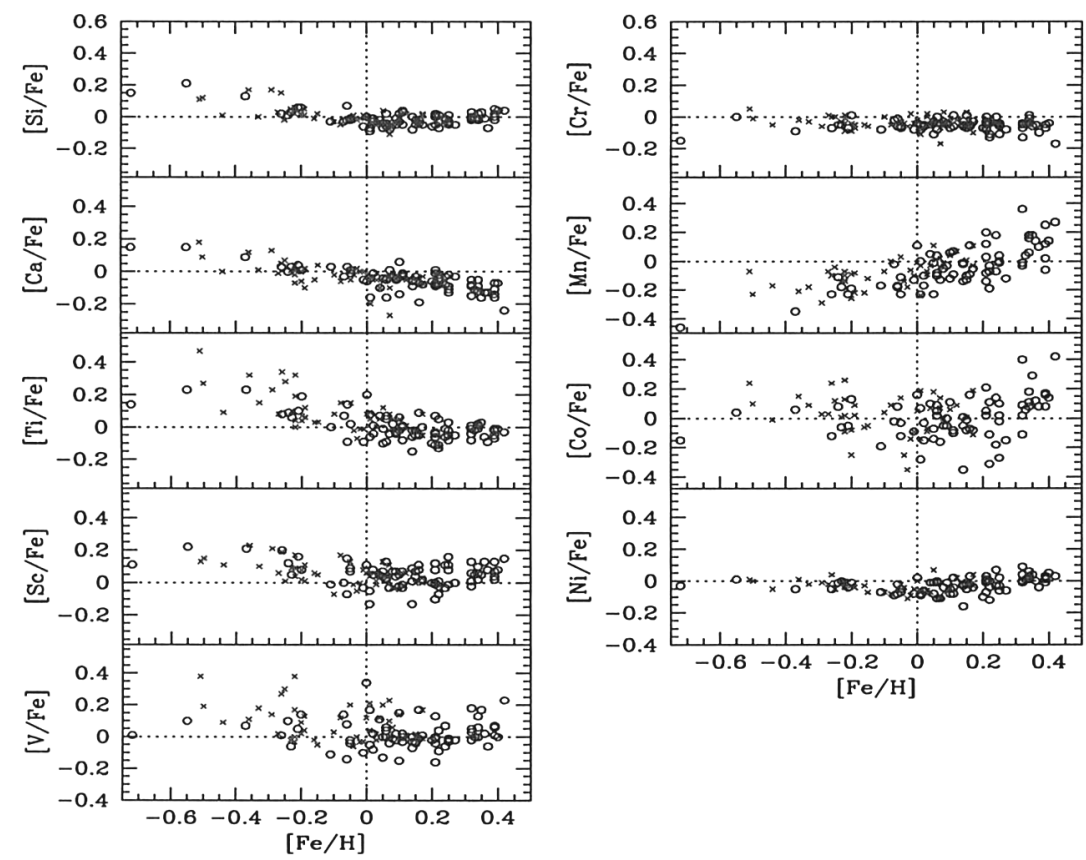

Figure 5. $\quad[\mathrm{X} / \mathrm{Fe}]$ vs $[\mathrm{Fe} / \mathrm{H}]$ plots for several $\alpha$ - and Fe-group elements. The crosses represent the comparison-sample stars, while the open circles denote the planet-hosting stars. From Bodaghee et al. (2003).

similar trends for $\mathrm{O}, \mathrm{Si}$ and all the other $\alpha$-elements (Tsujimoto et al.1995). Why $\mathrm{C}$ and $\mathrm{N}$ should have a different behaviour at high metallicities, both being volatiles and having similar production sites in the Galaxy? There can be three reasons for these anomalies; a) models of Galactic chemical evolution are very uncertain at high metallicities, $b$ ) abundance trends in metal-rich stars are affected by the presence of planets and c) abundance analysis of metal-rich stars is not reliable.

\subsection{Astrobiology}

Possible explanations for the abundance anomalies or the correlation between $[\mathrm{Fe} / \mathrm{H}]$ and the presence of giant planets have certain implications for astrobiology and even SETI. Already in the 1960's Drake (1965) and Shklovski (1966) independently proposed a method which extraterrestrial intelligent civilizations could employ in order to announce their existence. They could add some shortlived isotope(s) into the atmosphere of their "Sun" with the hope that possible observers would detect the absorption spectral lines of that element and realize their artificial origin. The amount of matter required to produce observable absorption lines of some rare elements/isotopes is not that large and any developed civilization should be able to handle this task.

The possible correlation between $[\mathrm{Fe} / \mathrm{H}]$ and the formation of giant planets may have an impact on the formation of terrestrial planets and their habitabil- 
ity via a shielding effect. Terrestrial planet formation should strongly depend on metallicity as well. A giant planet can relocate planetesimals and comets via scattering into the inner region of the planetary system. This process can protect inner habitable terrestrial planets against the impacts of the comets. Numerical simulations (Ida, Junko \& Lin 2001) show that a planet with large semi-major axis and/or large mass may eject planetesimals and prevent pollution of metals onto the host star. These authors proposed that the habitability may be regulated by a giant planet(s) since the shielding effect does not only inhibit impacts onto the host star but also prevents inner terrestrial planets from being impacted by cometary bodies.

The Earth contains a generous amount of all the stable and quasi-stable elements present in the Universe. The abundances of elements in the Sun and on the primitive Earth was suited to the creation and evolution of plants and living beings. Even the rather rare elements, those especially suited to the extraction of energy, using nuclear processes (like $\mathrm{U}$ and $\mathrm{Th}$ ) are present on the Earth in suitable concentration for the development of life. It is well known that the rock masses that form the external part of the Earth have been in rapid motion for hundreds of millions of years. This motion is so rapid that $2 / 3$ of the crust has been recycled into the Earth's mantle in the last 200 million years. Many geological processes (such as volcanic activity, drift of continents, iron catastrophe) in the lithosphere result from the heat released by the radioactive elements, mostly Thorium and Uranium. Active volcanoes are a source of many volatile compounds (water, methane, carbon oxides, etc.) which accumulated with time and formed the primitive gaseous atmosphere and, somewhat later, the liquid hydrosphere.

The above may suggest that there are certain chemical and physical preconditions for mankind's evolution. These preconditions (or "initial conditions"), depend on the chemical composition and evolution of the protoplanetary matter. Looking back, we can now see how the products of supernova explosions many billion years ago have influenced the life on our planet. Abundance studies in stars with exoplanets may help us to understand the chemical evolution of the planetary systems and learn about the evolution of life in the Universe.

Acknowledgments. I would like to thank my colleagues Nuno Santos, Michel Mayor, Rafael Rebolo, Alexandra Ecuvillon, Alister Graham and Ramón García López for many useful discussions and their comments on the text.

\section{References}

Bensby, T., Feltzing, S., \& Lundstrm, I 2003, in Carnegie Obs. Ap. Ser. Vol. 4, Origin and Evolution of the Elements, ed. McWilliam, A. \& Rauch, M.

Bodaghee, A., Santos, N. C., Israelian, G., \& Mayor, M. 2003, A\&A, 404, 715

Chen, Y. Q., Nissen, P. E., Zhao, G., Zhang, H. W., Benoni, T. A\&AS, 141, 491

Chen, Y. Q., Nissen, P. E., Benoni, T. \& Zhao, G. 2001, A\&A, 371, 943

Deliyannis, C. P., Demarque, P. \& Kawaler, S. 1990, ApJS, 73, 21

Drake, F. 1965, in Current Aspects of Exobiology. Oxford:Pergamon, 323 
Ecuvillon, A., Israelian, G., Santos, N. C. et al. 2004, in Proc. IAU Symp. 219, Stars as Suns: Activity, Evolution \& Planets, (A. K. Dupree \& A. O. Benz, eds.), CD-788

Edvardsson, B., Andersen, J., Gustafsson, B., et al. 1993, A\&A, 275, 101

Feltzing, S., \& Gustaffson, B. 1998, A\&AS, 129, 237

Forestini, M. 1994, A\&A, 285473

García López, R. J., \& Pérez de Taoro, M. R., 1998, A\&A, 334, 559

Gonzalez, G. 1997, MNRAS, 285, 403

Gonzalez, G., \& Laws, C. 2000, AJ, 119, 390

Gonzalez, G., Laws, C., Sudhi, T., \& Reddy, B. E. 2001, AJ, 121, 432

Gustafsson, B., Karlsson, T., Olsson, E., Edvardsson, B., \& Ryde, N. 1999, A\&A, 342, 426

Ida, S., Junko, K. \& Lin, D. N. C. 2001, A Symplectic Odyssey. Proc. of the 33rd Symp. on Celestial Mechanics, eds. E. Kokubo, T. Ito, and H. Arakida. Mitaka, Tokyo, Japan: NAO, 110

Israelian, G., Santos, N. C., Mayor, M., \& Rebolo, R. 2001, Nature, 411,163

Israelian, G., Santos, N. C., Mayor, M., \& Rebolo, R. 2003, A\&A, 405, 753

Israelian, G., Santos, N. C., Mayor, M., \& Rebolo, R. 2004, A\&A, 414, 601

Laws, C., Gonzalez, G., Walker, K. M. et al. 2003, AJ, 125, 2664

Mayor, M. \& Queloz, D. 1995, Nature, 378, 355

Murray, N., \& Chaboyer, B. 2002, ApJ, 566, 442

Nissen, P. E., \& Edvardsson, B. 1992, A\&A, 261, 255

Pinsonneault, M. H., DePoy, D. L., \& Coffee, M. 2001, ApJ, 556, L59

Pollack, J. B., Hubickyj, O., Bodenheimer, P. et al., 1996, Icarus, 124, 62

Reddy, B., Lambert, D., Laws, C., et al. 2002, MNRAS, 335, 1005

Ryan, S. 2000, MNRAS, 316, L35

Sadakane, K., Ohkubo, M., Takeda,Y. et al. 2002 PASJ, 54, 911

Sadakane, K. et al. 2003, in IAU Symposium 219, Stars as Suns: Activity, Evolution and Planets, Poster Abstract IAU 01801

Sandquist E.L., Dokter J. J., Lin D.N.C. \& Mardling, R. 2002, ApJ, 572, 1012

Santos, N. C., Israelian, G., \& Mayor, M. 2000, A\&A, 363, 228

Santos, N. C., Israelian, G., \& Mayor, M. 2001, A\&A, 373, 1019

Santos, N. C., García López, R.J., Israelian, G. et al. 2002, A\&A, 386, 1028

Santos, N. C., Israelian, G., Mayor, M., Udry, S. \& Rebolo, R. 2003a, A\&A, 398, 363

Santos, N. C., Israelian, G., \& Mayor, M. 2003b, A\&A, submitted

Shklovski, I. 1966, in Intelligent Life in the Universe, N.Y., Deli, 1966, p. 406

Smith, V. V., Cuhna, K., \& Lazzaro, D. 2001, AJ, 121, 3207

Takeda, Y., Sato, B., Kambe, E., Aoki, W. et al. 2001, PASJ, 53, 1211

Tsujimoto, T., Yoshii, Y., Nomoto, K., Shigeyama, T. 1995, A\&A, 302, 704

Udry S., Mayor M., Naef D., et al. 2002, A\&A, 356, 590

Zucker, S. \& Mazeh, T. 2002, ApJ, 568, L113 